\title{
Effect of porosity and hydrostatic pressure on water absorption in a semicrystalline fluoropolymer
}

\author{
Camilo Castro López ${ }^{1,2} \cdot$ Xavier Lefebvre $^{1} \cdot$ Nadège Brusselle-Dupend $^{1}$ • \\ Marie-Hélène Klopffer ${ }^{1}$ - Laurent Cangémi ${ }^{1}$ - Sylvie Castagnet ${ }^{2}$. \\ Jean-Claude Grandidier ${ }^{2}$
}

\begin{abstract}
This paper discusses the effect of porosity and hydrostatic pressure on diffusion kinetics and equilibrium water uptake in a semicrystalline fluoropolymer. Water sorption experiments at atmospheric pressure and under water pressures up to $250 \mathrm{MPa}$ were carried out during 18 months at $40{ }^{\circ} \mathrm{C}$ on reference and porous samples. Porosity of samples was induced due to a cavitation process occurring at the highest triaxiality area of waisted and notched specimens during tensile tests. Water uptake was found to be very sensitive to porosity, showing an increase in samples with a high void fraction. On the other hand, water content decreased with increasing pressure suggesting a compaction of the porous space in which water can be stored. Two models describing this water uptake behaviour were considered. The first is a classical model which assumes that sorption occurs only by diffusion following Fick's law. Fick's model was found to be in agreement with the experimental results. A "Langmuir-type" sorption model was also proposed to describe water uptake in porous samples, considering a two-phase water transport mechanism: one portion of the absorbed water diffuses through the polymer matrix and the other portion is stored in voids. This model was implemented in a user subroutine using ABAQUS ${ }^{\mathrm{TM}}$ software and simulations were confronted to experimental sorption curves showing satisfactory agreements. The potential of the Langmuir-type sorption model resides on its availability to be coupled to a
\end{abstract}

Xavier Lefebvre

xavier.lefebvre@ifpen.fr

1 IFP Energies nouvelles, 1 et 4 avenue de Bois-Préau, 92852 Rueil-Malmaison, France

2 Institut PPRIME, ENSMA, 86360 Chasseneuil-du-Poitou, France poro-mechanical model, in order to improve the understanding of coupling between the mechanical behaviour and water sorption mechanism in a porous polymer.

\section{Introduction}

In several areas of engineering and industry, semicrystalline polymers are often used as protective coatings because of their barrier properties. Depending on the nature of the polymer, the presence of water in its microstructure can have several effects on its mechanical behaviour such as swelling, plasticization or ageing. When exposed a long time in a humid environment, characterization of water transport mechanisms in these materials is well worth investigating.

Transport properties of water can be affected by the microstructure and/or the mechanical loading of the polymer. Little is known in the literature about the effect of porosity or hydrostatic pressure on the water sorption of semicrystalline polymers.

Some authors have studied the impact of hydrostatic pressure on water absorption in thermosetting resins and composites. As an example, sorption tests under pressure performed by Filyanov et al. [1] in epoxy resins showed a decrease of water content when samples were immersed under pressures up to $100 \mathrm{MPa}$. However, other authors reported no effects on water absorption at 25 and $50 \mathrm{MPa}$ of pressure in a cured epoxy resin [2], or at $10 \mathrm{MPa}$ of pressure in polyester and vinyl ester resins [3, 4]. Some polyester resins showed a slight decrease in water sorption under pressures up to $75 \mathrm{MPa}$ when immersed at low temperature $\left(5^{\circ} \mathrm{C}\right)$.

Other authors have also noted an increase in moisture absorption at hydrostatic pressure in glass fibre epoxy 
composites [2, 5] and in glass fibre-reinforced polyester composites [6].

In a study performed on a polyurethane containing glass microspheres, water uptake increased with hydrostatic pressure due to progressive collapse of the glass bubbles [2].

Several works have focused on the study of water sorption in fibre-reinforced composites, in which the effect of external stress on water content depends on several aspects such as the nature of the matrix, fibre content and fibre orientation [7, 8]. In some analytical models, free volume change due to external stress has been used to describe the water transport behaviour in the polymer matrix [9-13].

Impact of porosity on water uptake has been briefly studied on epoxy resin composites [14-17] where the volume of voids has clearly an important effect by increasing the amount of water absorbed.

Semicrystalline fluoropolymers are often used as protective coating material in wet environments, which make it important to have a good knowledge of its sorption characteristics to assess the long-term properties of polymer structures. Only a few works have reported water sorption in this kind of polymers $[18,19]$ and none have considered hydrostatic pressure or porosity effects.

The present study aimed to characterize the influence of both the microstructural state of the material and its hydrostatic confinement, on the water absorption mechanism, in a fluoropolymer grade. For this purpose, gravimetric experiments were performed to study the sorption kinetics of samples with several void fractions and under high hydrostatic pressure. Subsequently, two sorption models were discussed to the interpretation of the water transport behaviour observed from experiments.

\section{Experimental procedure}

\section{Preparation of specimens}

Specimens were obtained from the core of an extruded commercial grade of a semicrystalline fluoropolymer. In order to study the porosity effect in sorption tests, samples containing three different initial porosity levels were used for experiments. Cylindrical specimens with a $2.5 \mathrm{~mm}$ radius were manufactured for sorption tests on reference samples. Specimens of different initial geometries were used to induce porosity due to tensile tests. Large strains in tension induce void formation in many semicrystalline polymers that are sensitive to multiaxial stress states. The level of porosity developed during tensile tests increases with the stress triaxiality ratio, which is linked to the initial notch radius of specimens [20]. The lower the notch radius,

the higher the stress triaxiality effect that favours the cavitation process [21-24].

To obtain samples with different porosity levels, tensile tests were carried out in waisted and notched round bars specimens (Fig. 1) at room temperature, which is above the glass transition temperature of the studied material. Tensile test were performed in an Instron-type mechanical machine with a strain rate of $5 \times 10^{-3} \mathrm{~s}^{-1}$.

After interrupting tensile tests up to strain of about $75 \%$ for $\mathrm{WB}$ and $85 \%$ for $\mathrm{NB}$, the porous zone (homogenous whitened area) was removed from the unloaded specimens (Fig. 2a), avoiding the neck shoulder to ensure homogeneity in the void fraction of samples as previously observed from X-ray microtomography analysis in notched specimens after unloading [22]. The void fraction (about $20 \%$ ) is globally uniform in the area of constant diameter (dotted square in Fig. 2b). Profiles of porosity in the longitudinal and radial cross sections were obtained from image processing of this area. The radial cross section showed a gradient of porosity due to a densification near to the surface, while longitudinal distribution of porosity remained uniform. The effective void fraction of samples was determined through density measurements based on Archimedes' principle (see "Density determination of samples" section). Samples were then immersed in water at $40{ }^{\circ} \mathrm{C}$ for 18 months, with or without hydrostatic pressure, for the sorption experiment (see "Water sorption test" section).

\section{Density determination of samples}

The "apparent" density refers to the weight per volume unit of sample, including porosity in the material. Following the Archimedes' principle, the density of the solid sample is determined thanks to a liquid of known density (water in this case). The sample is weighed in air and water

(a)

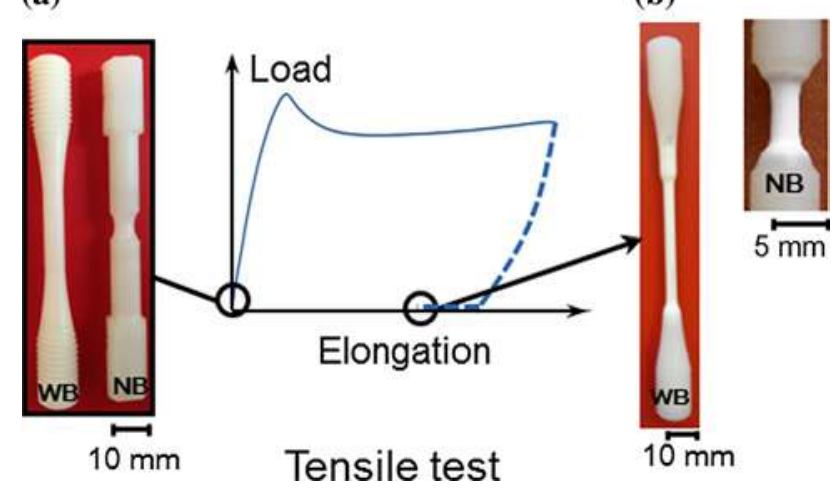

Fig. 1 a Waisted bar (WB) with $5 \mathrm{~mm}$ cross-section diameter and notched bar (NB) with an initial $5.5 \mathrm{~mm}$ notch radius. b Specimens after interrupted tensile tests 

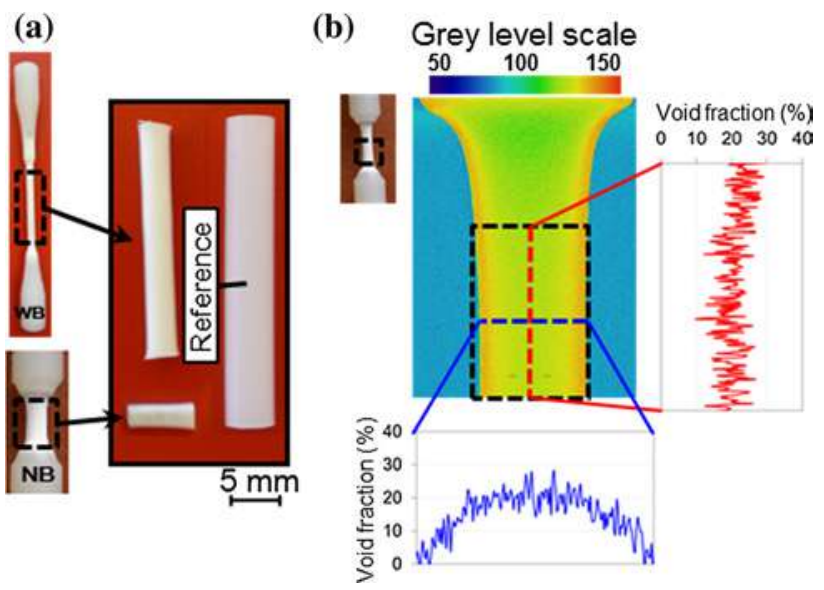

environment, causing a significant initial increase in density (reduction of porosity) which stabilized afterwards. As an illustration, Fig. 3 shows the evolution of density of a porous sample with a measured void fraction at room temperature of $7 \%$. After $36 \mathrm{~h}$ at $40{ }^{\circ} \mathrm{C}$, the increase in density reduced void fraction at $5 \%$. Even after 1 month of heating, void fraction remained stable around $5 \%$.

Thus, due to the temperature effect on density, the initial void fraction $f_{0}$ of samples was measured after a few hours of heating at $40{ }^{\circ} \mathrm{C}$ : initial void fraction of samples obtained from waisted and notched specimens was then evaluated at about 4.3 and $14 \%$, respectively.

\section{Water sorption test}

Fig. 2 a Samples with three initial porosity levels used for sorption tests. b Longitudinal cross section and porosity profiles of an unloaded notched specimen with an initial $5.5 \mathrm{~mm}$ notch radius obtained from X-ray microtomography according to [22]

successively and density is calculated from the two weighings as follows:

$\rho_{\text {sample }}=\frac{m_{\text {sample }}^{\mathrm{a}}}{m_{\text {sample }}^{\mathrm{a}}-m_{\text {sample }}^{\mathrm{w}}}\left(\rho_{\mathrm{w}}-\rho_{\mathrm{a}}\right)+\rho_{\mathrm{a}}$,

where $m_{\text {sample }}^{\mathrm{a}}$ is the sample mass in air, $m_{\text {sample }}^{\mathrm{w}}$ is the sample mass in water, and $\rho_{\mathrm{w}}$ and $\rho_{\mathrm{a}}$ are, respectively, the water and air densities.

Void fraction $f$ can be obtained from density measurements as follows:

$f=1-\frac{\rho_{\text {porous }}}{\rho_{\text {reference }}}$,

where $\rho_{\text {porous }}$ and $\rho_{\text {reference }}$ are the porous and reference samples densities.

Since porous samples were immersed into water heated at $40{ }^{\circ} \mathrm{C}$, it was necessary to take into account the relaxation effect due to temperature. It was found that porous samples relaxed when heated at $40{ }^{\circ} \mathrm{C}$ in a dry

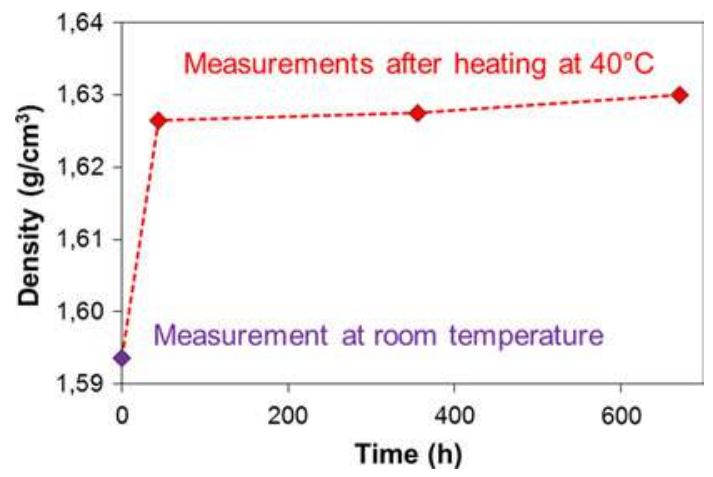

Fig. 3 Temperature effect on density of a porous sample

The kinetics of water sorption in reference and porous samples was studied at atmospheric pressure and under hydrostatic pressure at 100 and $250 \mathrm{MPa}$. Samples were immersed in water heated to $40{ }^{\circ} \mathrm{C}$ and water uptake was monitored by gravimetric measurements. When removed from the water, the surface of the specimens was wiped with a soft tissue before weighing, in order to remove the water trapped at the surface.

A Mettler Toledo XS205 analytical balance with $40 \mu \mathrm{g}$ accuracy was used to weigh the samples immersed at atmospheric pressure.

For gravimetric experiments under high pressure, samples were immersed in autoclaves with chambers of $6 \mathrm{~mm}$ diameter and $150 \mathrm{~mm}$ length. The pressurization and depressurization rate was $100 \mathrm{MPa} / \mathrm{h}$. Measures of mass were performed using a Mettler Toledo MT5 analytical balance with $12 \mu \mathrm{g}$ accuracy.

Weight uptake percentage WU\% in samples was determined from Eq. (3):

$\mathrm{WU} \%=\frac{m_{t}-m_{0}}{m_{0}} \times 100$,

where $m_{0}$ is the initial mass of the dry sample and $m_{t}$ is the mass of the wet sample. Samples were duplicated and results of water uptake were found to be repeatable.

\section{Experimental results}

\section{Effect of water uptake in "apparent" density at atmospheric pressure}

In order to improve the understanding of water sorption in the studied fluoropolymer, the effect of water uptake on the density of the samples was monitored at atmospheric pressure.

There are two limiting hypotheses to explain how polymer density changes due to water absorption: the first 
one assumes that the volume of the sample does not change upon water sorption, whereas the second assumes volume additivity [25]. In the constant volume case, no dimensional change results from water sorption and density is given by Eq. (4):

$\rho_{\text {sample }}^{\mathrm{w}}=\frac{\rho_{\text {sample }}}{1-Y_{w}}$.

In the volume additivity case, the volume sample increases by the volume of water absorbed, and the density is calculated from Eq. (5):

$\rho_{\text {sample }}^{\mathrm{w}}=\frac{\left(1+Y_{\mathrm{w}}\right) \rho_{\mathrm{w}} \rho_{\text {sample }}}{\rho_{\mathrm{w}}+Y_{\mathrm{w}} \rho_{\text {sample }}}$,

where $\rho_{\text {sample }}^{\mathrm{w}}$ is the wet sample density, $\rho_{\text {sample }}$ is the initial density of the dry sample, and $Y_{\mathrm{w}}$ is the water mass fraction absorbed by the sample, defined as $m_{\mathrm{w}} / m_{0}, m_{\mathrm{w}}$ being the mass of water and $m_{0}$ the initial mass of the sample.

The density of samples at atmospheric pressure was measured periodically during the sorption test. In Fig. 4, it was plotted as a function of the water uptake and compared with the curves obtained from the limiting cases of density variation presented above.

In reference samples, experimental data seemed to follow the volume additivity model, indicating that water sorption induced swelling. The very low water uptake of these samples could be the cause of dispersion observed on experimental data. On the other hand, experimental data of porous samples followed the model of constant volume suggesting that water absorbed by the samples filled the voids without any change in sample dimensions.

\section{Porosity effect on water sorption}

Weight uptake due to water sorption was plotted against the square root of time, normalized by the radius of the sample (Fig. 5), since this is a representation commonly used for comparison with classical Fick's diffusion theory [26]. The normalization of the axis enables to take into account geometrical effects due to differences between radii for samples with various void fractions (Fig. 2a).

It was very clear that the void fraction had a significant effect on the amount of water absorbed, which was considerably low in reference samples. In fact, it seemed that water content reached the equilibrium in reference samples after 100 days $\left(20 \mathrm{~h}^{1 / 2} / \mathrm{mm}\right.$ in Fig. 5), while water absorption in porous samples continued to increase almost linearly. Porosity in samples was induced by a cavitation process due to tensile tests, which could involve growth of cavities and a percolation effect. This latter phenomenon in the porous structure could also favour water uptake during the sorption test.
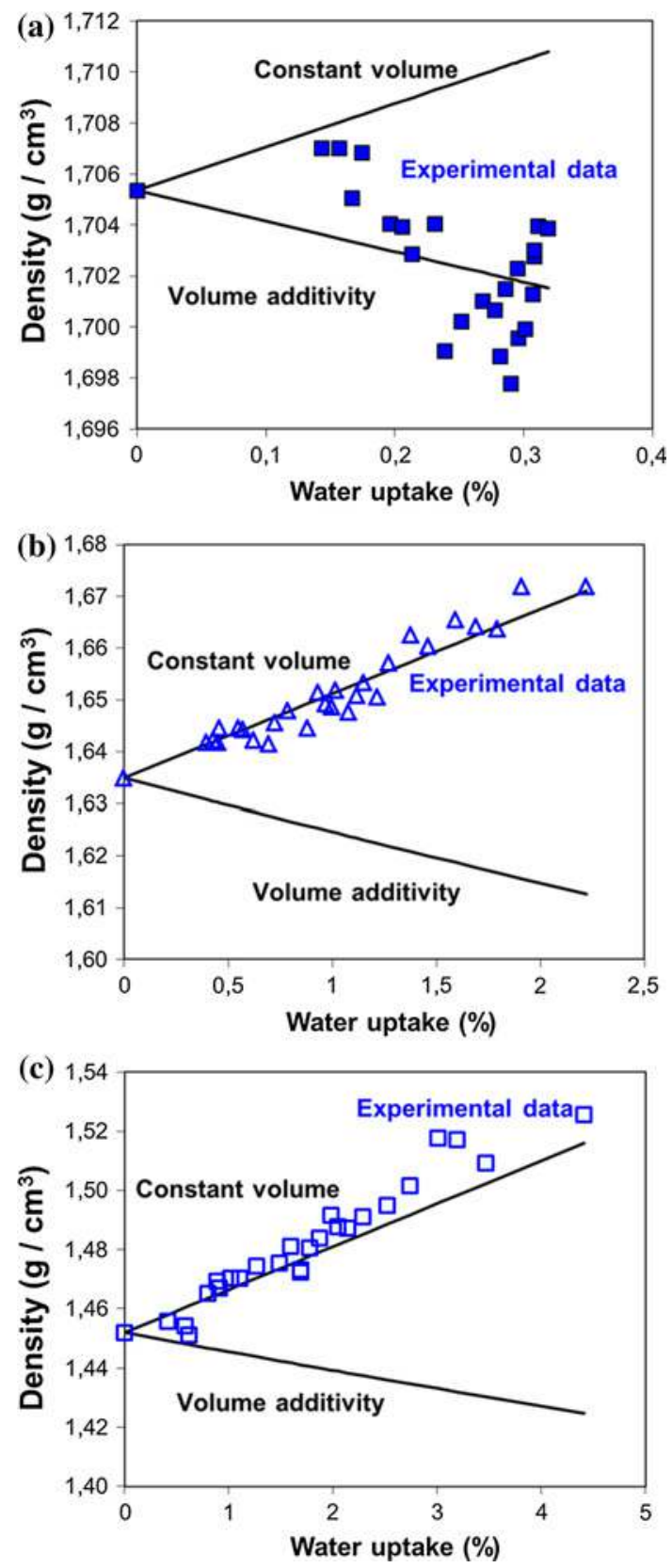

Fig. 4 Effect of water sorption on the apparent density of samples at atmospheric pressure. a Reference samples, b porous samples with $4.3 \%$ of void fraction, $\mathbf{c}$ porous samples with $14 \%$ of void fraction

Similar effects of porosity on water sorption had also been reported in glass/epoxy composites [15] and in carbon-reinforced laminates $[14,17]$.

\section{Hydrostatic pressure effects}

\section{Reference samples}

Water sorption tests were carried out on reference specimens immersed at atmospheric pressure, 100 and $250 \mathrm{MPa}$. 


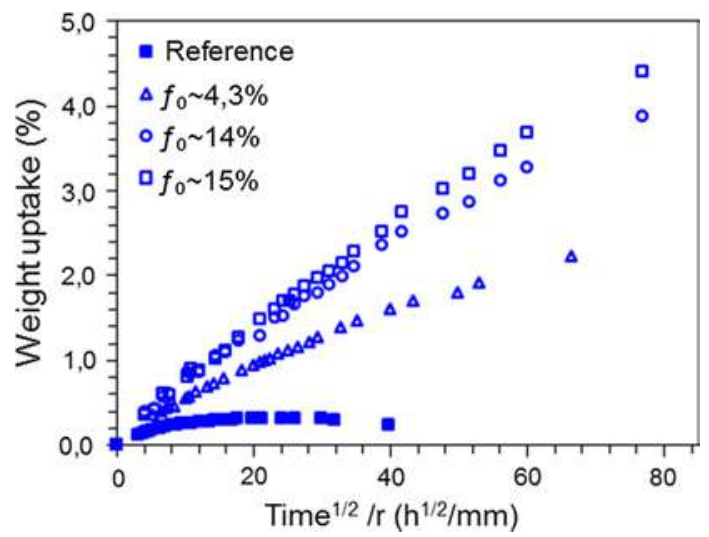

Fig. 5 Weight uptake at atmospheric pressure in samples with different initial void fractions

Results from these sorption experiments are plotted in Fig. 6. Water uptake was found to decrease with increasing pressure. At atmospheric pressure, the gain in weight of the sample seemed to have reached equilibrium around 100 days $\left(50 \mathrm{~h}^{1 / 2}\right)$ and a weight loss started around 200 days $\left(70 \mathrm{~h}^{1 / 2}\right)$. This decrease could be related to a loss of additives initially present in the polymer (e.g. plasticizer), due to the long-term exposure in water at $40{ }^{\circ} \mathrm{C}$. Here, the competition between water uptake and loss of additives was revealed because water uptake could be close to the saturation level. However, samples under hydrostatic pressure did not show the weight loss. A hypothesis to explain that fact involved the volume of water surrounding the immersed samples. Tests under high pressure were performed in autoclaves where the chamber used for confining had a small volume, whereas samples at atmospheric pressure were immersed in a much bigger recipient, where the volume of water around the samples was significantly larger. When samples were immersed in a large volume of water (around 200 times larger than the specimen volume), the additives concentration in water remained very low despite its desorption from the material. Consequently, this

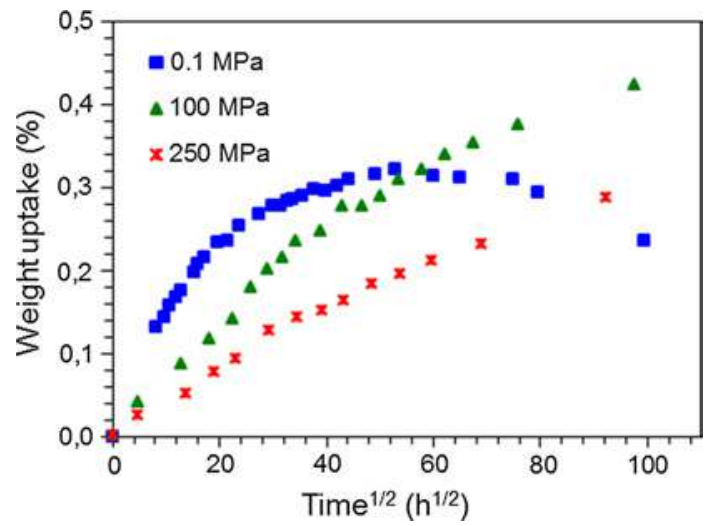

Fig. 6 Weight uptake at atmospheric pressure, 100 and $250 \mathrm{MPa}$ of hydrostatic pressure on reference samples higher concentration gradient of additives favours its migration from sample to water.

\section{Porous samples}

Sorption tests on porous samples were performed under the same conditions of temperature and pressure as reference samples. Water sorption in porous samples was also found to decrease with increasing hydrostatic pressure (Figs. 7, 8). This decrease could be related to a closing of the voids under hydrostatic compression. Decrease of water uptake with $100 \mathrm{MPa}$ of applied pressure was larger in samples with $14 \%$ of initial void fraction than those with $4.3 \%$. This result suggested that compressibility was more significant in porous samples with a higher void fraction.

Besides, there was no visible weight loss in porous samples as observed in the reference samples at atmospheric pressure. In porous samples, the amount of water uptake could be large enough to lead the weight evolution and hide the loss of additives.

In order to explain the water sorption behaviour on reference and porous samples observed above, two theoretical sorption models are discussed.

\section{Water sorption modelling}

Two approaches of sorption modelling were studied to describe the water transport behaviour of samples. Initially, there was an interest in determining if water diffusion could be described by classical diffusion theory (Fick's law), since this is a commonly used model for diffusion in polymeric systems. In addition, to go further into sorption modelling for porous samples, a Langmuir-type model was proposed assuming a sorption mechanism linked to the presence of voids. The two models are successively considered here.

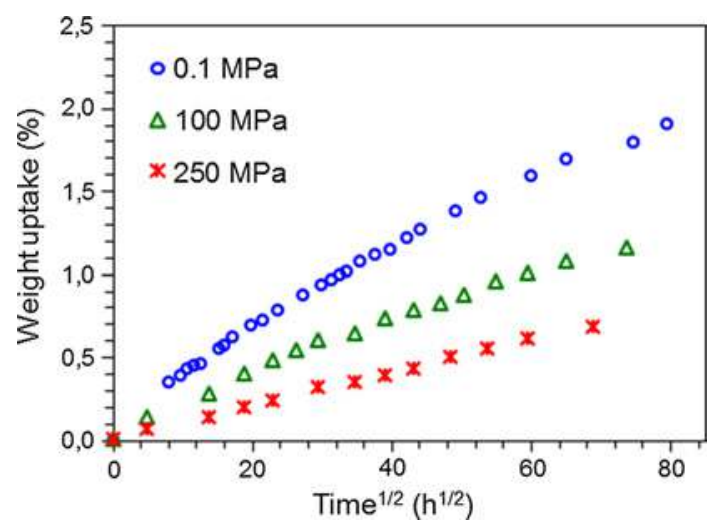

Fig. 7 Weight uptake at atmospheric pressure, 100 and $250 \mathrm{MPa}$ of hydrostatic pressure on samples with $4.3 \%$ of initial void fraction 


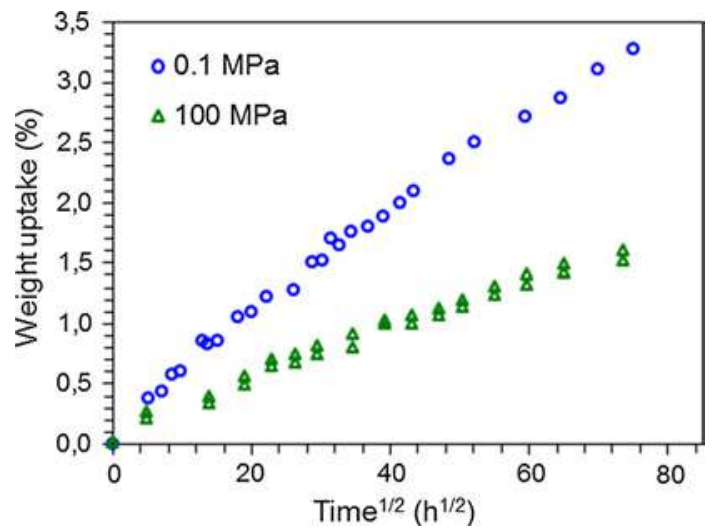

Fig. 8 Weight uptake at atmospheric pressure and $100 \mathrm{MPa}$ of hydrostatic pressure on samples with $14 \%$ of initial void fraction

\section{Fickian description of water sorption}

According to the classical diffusion theory, the diffusion coefficient $D$ could be identified from sorption curves, since the weight uptake is linear as a function of the square root time at the earlier stages (usually $m_{t} / m_{\infty} \leq 0.5$ where $m_{t}$ is the mass uptake at time $t$, and $m_{\infty}$ is the mass uptake at equilibrium) [26]. From Figs. 6, 7 and 8, it was clear that water uptake at equilibrium (or solubility) has not been reached yet in all the sample thicknesses, even after a longterm exposure to water (more than 1 year). However, linearity in water uptake could be observed at least up to $50 \mathrm{~h}^{1 / 2}$. Even if solubility was unknown, its value could be estimated to identify a diffusion coefficient by fitting theoretical curves to experimental water uptake curves. Diffusion coefficient for each tested sample was identified according to Fick's law applied to a cylindrical geometry [27].

Identified solubility and diffusion coefficients are plotted as a function of void fraction in Fig. 9. The samples with high void fraction showed the smallest diffusion coefficient and the highest solubility. Both solubility and diffusion coefficient seemed to have a non-linear evolution.
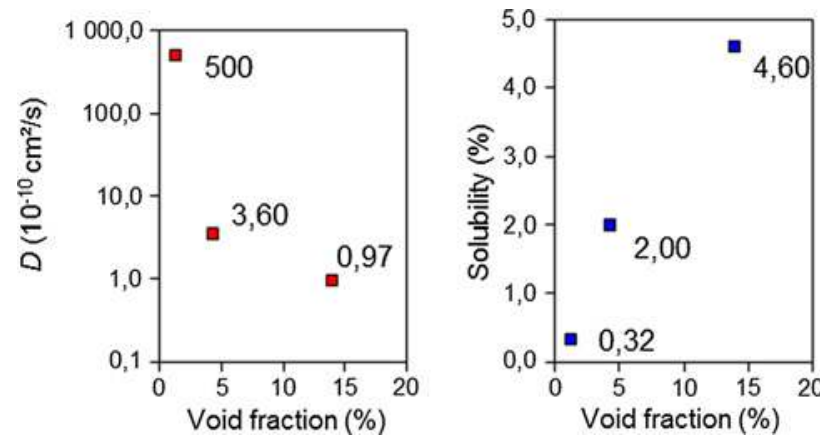

Fig. 9 Diffusion coefficient and solubility as a function of void fraction for samples immersed in water at atmospheric pressure
The same sorption parameters are plotted as a function of hydrostatic pressure in Fig. 10. According to experimental observations, it was assumed that water uptake at equilibrium was reduced by the effect of increasing hydrostatic pressure. Except for reference samples immersed at atmospheric pressure, the diffusion coefficient did not seem to be affected by the applied pressure.

A non-pressure dependence of the diffusion coefficient has previously been reported in epoxy resins $[1,4,28]$.

Comparisons between experimental water uptake curves and Fick's theoretical model are shown in Fig. 11 for reference samples and in Fig. 12 for porous samples.

For all porous samples and for reference samples under high pressure, a very satisfactory agreement is observed between experiments and the Fickian model. Agreement of the model with experiments for reference samples at atmospheric pressure was satisfactory only up to 100 days $\left(20 \mathrm{~h}^{1 / 2} / \mathrm{mm}\right.$ in Fig. 11). The weight loss observed in those samples was not taken into account for modelling.

A particular behaviour was observed in reference samples immersed at atmospheric pressure, which is the only case where $D$ was found to be significantly higher than those immersed under high pressure (100 and $250 \mathrm{MPa}$ ). Here, $D$ was determined based on the apparent equilibrium water uptake observed around $50 \mathrm{~h}^{1 / 2}$.

As previously suggested (see "Hydrostatic pressure effects" section), a possible loss of additives could be distorting the "real" water uptake behaviour on reference samples immersed at atmospheric pressure. Considering that statement, a new solubility was estimated for reference samples at atmospheric pressure assuming $D$ as independent on the applied hydrostatic pressure. This assumption is coherent with results obtained for all other samples (Fig. 10). A higher solubility was then identified, showing a decreasing evolution of solubility with pressure, such as observed in porous samples (Fig. 13).

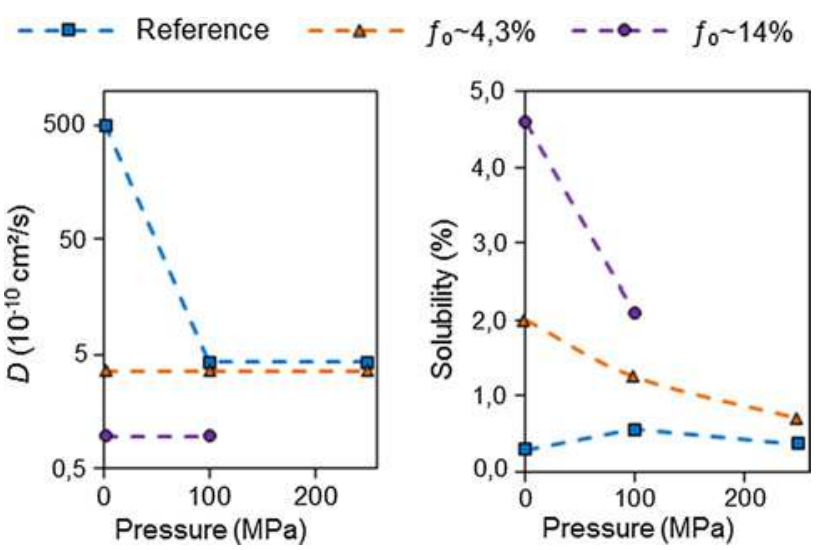

Fig. 10 Diffusion coefficient and solubility as a function of water pressure 


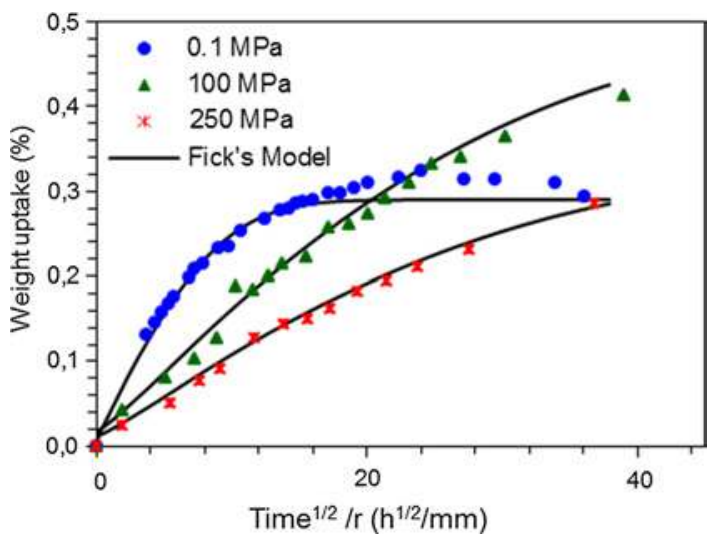

Fig. 11 Description of water uptake of reference samples with classical Fick's law

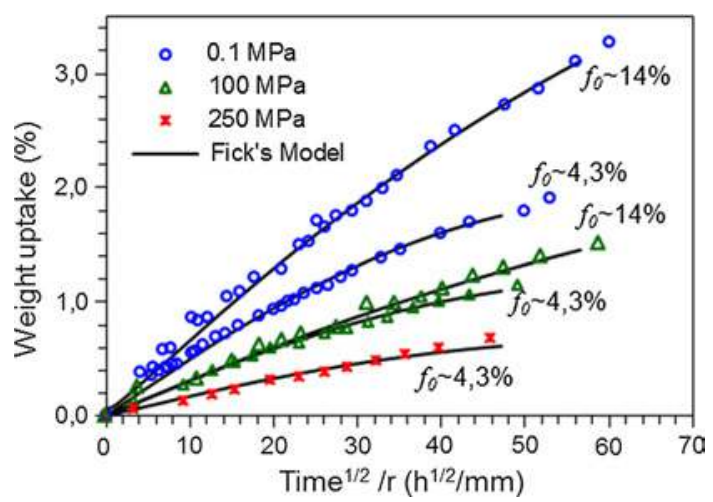

Fig. 12 Description of water uptake of porous samples with classical Fick's law

The theoretical Fick curve of water uptake obtained under this assumption is plotted with a dotted line in Fig. 14. In this case, experimental data would be the result
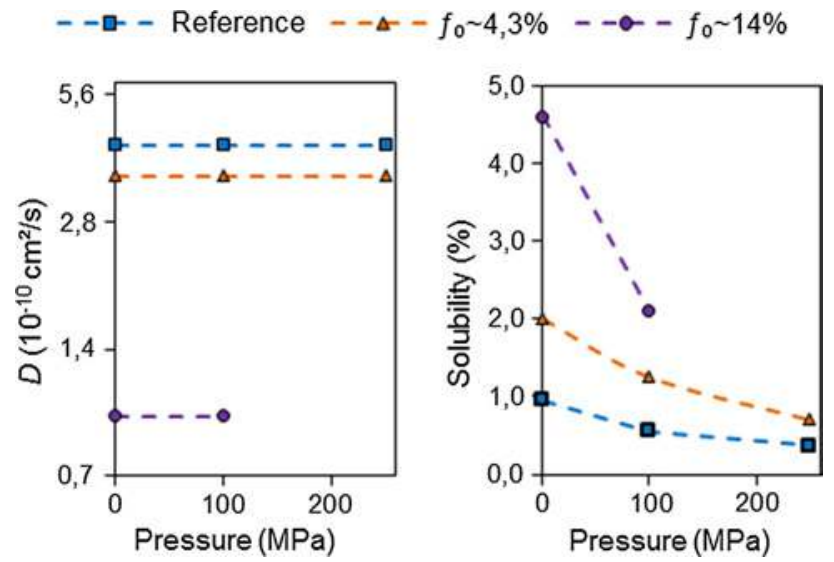

Fig. 13 Diffusion coefficient $D$ and solubility as a function of water pressure. Solubility on reference samples at atmospheric pressure was estimated assuming $D$ independent on pressure of the weight gain by water uptake, in competition with the weight loss brought by the extraction of the additives. Accordingly with this interpretation, the gap between the theoretical Fick curve and experimental data could correspond to the loss of additives.

Nevertheless, further experiments are necessary to confirm this hypothesis. For example, if sorption tests were performed at atmospheric pressure in a volume of water as small as in experiments under pressure, the concentration gradient of additives between the sample and the water should be very small, avoiding migration of additives from samples to water. Thus, it could be possible to discriminate a possible effect of additives in the sorption experiments at atmospheric pressure. Another experiment could be to determine the additives content before and after sorption tests; however, the weight loss is too small to be accurately quantified by thermogravimetric analysis for example.

From here on, hypothesis of loss of additives at atmospheric pressure in reference samples is adopted for further modelling. Thus, diffusion coefficient and solubility in reference samples refer to those identified in Fig. 13.

With the classical Fick's model, only diffusion coefficient $D$ and solubility were necessary to describe the global sorption behaviour of the material, which simplified modelling. Still, it was found that the porous structure of the polymer significantly affected the water sorption behaviour of samples. Fick's model gives a global description of water uptake without linking it to porosity. To go further in the water uptake modelling of porous samples, a Langmuirtype sorption model was then proposed by considering a sorption mechanism where water penetrating the polymer diffuses through the matrix and fills the voids. This kind of approach enables to directly link water sorption to the void fraction of samples.

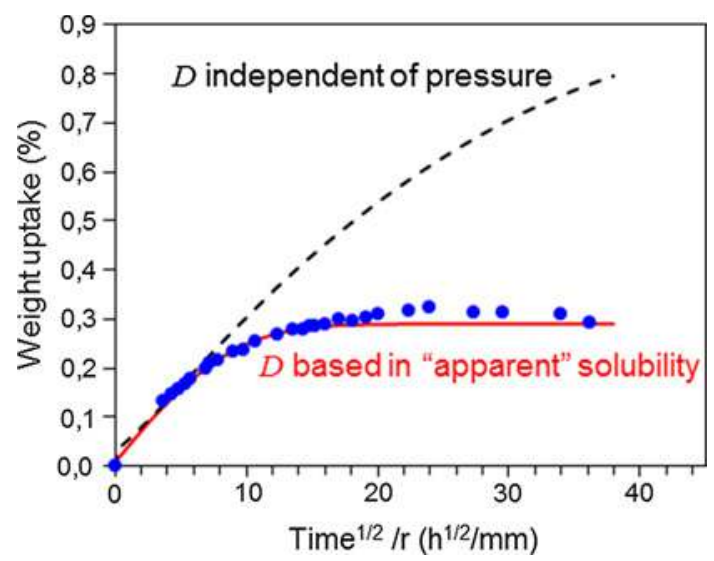

Fig. 14 Fick's model fitting on water sorption curves for reference samples at atmospheric pressure. Dotted curve hypothesis of modelling without loss of additives 


\section{Consideration of cavities in the sorption model}

The large amount of water in porous samples and the results shown in Fig. 4 suggested that the water penetrating the polymer could fill the volume of voids. To take this effect into account in the sorption model, it was assumed that the water absorbed by the porous polymer was divided into two parts: a free or mobile fraction diffusing through the polymer matrix and a bound fraction filling the voids.

The notion of free/bound water in sorption models has already been proposed to describe non-Fickian behaviour on epoxy resins and adhesives [29-31].

\section{Basis of the "Langmuir-type" sorption model}

Carter and Kibler [29] proposed a diffusion model socalled Langmuir-type because of its similarity to Langmuir adsorption. According to this model, anomalous sorption behaviour could be explained from the assumption that absorbed water existed in two phases (mobile and bound).

This approach suggests that bound water could become mobile (and vice versa) with certain probability in a unit time. Therefore, the process of diffusion of mobile water conforms to the classical Fick's theory, modified by source or sink terms (bound water). For the one-dimensional case, the molecular number densities $(N$ and $n)$ at position $z$ and time $t$ satisfied the coupled pair of equations:

$\frac{\partial n}{\partial t}=-D \frac{\partial^{2} n}{\partial z^{2}}-\frac{\partial N}{\partial t}$,

$\frac{\partial N}{\partial t}=\gamma n-\beta N$,

where $D$ is a constant coefficient of diffusion, $n$ represents the number of mobile molecules (per unit volume) with a probability $\gamma\left(\mathrm{s}^{-1}\right)$ to become bound and $N$ the number of bound molecules with a probability $\beta\left(\mathrm{s}^{-1}\right)$ to become mobile.

\section{Proposed sorption model for the porous polymer}

In the present work, authors proposed a model to describe the sorption behaviour of porous samples inspired from the Langmuir-type sorption model of Carter and Kibler. Here, the Fickian diffusion process is supposed to be disturbed by filling of voids, which act as a sink term modifying the transport kinetics.

Total water mass fraction in the sample was then considered to be the addition of "mobile" (in the matrix) and "bound" (in the cavities) water mass fractions, as depicted by Eq. (8):

$Y_{\mathrm{w}}=\frac{m_{\mathrm{w}}}{m_{0}}=Y_{\mathrm{w}}^{\mathrm{mat}}+Y_{\mathrm{w}}^{\mathrm{cav}}$.
Water mass fraction was defined as follows:

$Y_{\mathrm{w}}^{i}=\frac{m_{\mathrm{w}}^{i}}{m_{0}}$,

where $Y_{\mathrm{w}}^{i}$ was the water mass fraction in $i$ (i, being the matrix or the cavities), $m_{\mathrm{w}}^{i}$ was the mass of water in $i$ and $m_{0}$ was the initial mass of the sample.

Two equilibrium conditions were defined, related to the water content. The first condition was when the sample was completely dry and the second when the sample was fully saturated:

In the dry state, neither the matrix nor cavities contained water. Both mobile and bound water mass fractions were zero.

$Y_{\mathrm{w}}^{\mathrm{mat}}=Y_{\mathrm{w}}^{\mathrm{cav}}=0$.

In the wet equilibrium state, both the matrix and cavities were water saturated:

$Y_{\mathrm{w}}^{\mathrm{mat}}=Y_{w(\infty)}^{\mathrm{mat}} \quad$ and $\quad Y_{\mathrm{w}}^{\mathrm{cav}}=Y_{\mathrm{w}(\infty)}^{\mathrm{cav}}$.

The equilibrium water mass fraction in cavities $Y_{\mathrm{w}(\infty)}^{\mathrm{cav}}$ was expressed as the amount of water necessary to fill the volume of pores:

$Y_{\mathrm{w}(\infty)}^{\mathrm{cav}}=\frac{f \rho_{\mathrm{w}}}{\rho_{\mathrm{m}}(1-f)}$,

where $\rho_{\mathrm{w}}$ and $\rho_{\mathrm{m}}$ are, respectively, the water and the matrix density, and $f$ is the void fraction of the sample.

The water transport behaviour consisted of Fickian diffusion through the matrix modified by the presence of voids. Evolution of water mass fraction was expressed as follows:

$\frac{\partial Y_{\mathrm{w}}^{\mathrm{mat}}}{\partial t}=-D \operatorname{div} \nabla Y_{\mathrm{w}}^{\mathrm{mat}}-\alpha \frac{\partial Y_{\mathrm{w}}^{\mathrm{cav}}}{\partial t}$,

where $D$ is the classical Fickian diffusion coefficient of water in the matrix (reference material). The last term on the right side of Eq. (13) is considered as a "sink" and represents the water trapped into the voids.

As suggested in [15], voids provide water storage at a much higher concentration than in the matrix. Parameter $\alpha$ was introduced as a water transfer coefficient between the matrix and the voids, it represents the proportion in which the filling of voids affected diffusion in the matrix, and consequently global water uptake kinetics.

The kinetics of voids filling was described from the phenomenological expression given by Eq. (14) in which a characteristic time $\tau$ rules the filling rate of cavities with water. During immersion, water in cavities gradually increased towards saturation, fed by the water present in the matrix. Filling stopped when reaching saturation (wet equilibrium state). 
$\left.\frac{\partial Y_{\mathrm{w}}^{\mathrm{cav}}}{\partial t}=-\frac{1}{\tau} \quad Y_{\mathrm{w}}^{\mathrm{cav}}-Y_{\mathrm{w}(\infty)}^{\mathrm{cav}} \frac{Y_{\mathrm{w}}^{\mathrm{mat}}}{Y_{\mathrm{w}(\infty)}^{\mathrm{mat}}}\right)$.

Parameters $\alpha$ and $\tau$ needed to be identified by finding the best match between experimental curves and theoretical curves obtained with the proposed model.

By splitting the water mass fraction entering the polymer into two parts, it was necessary to determine the equilibrium water content of mobile and bounded water $\left(Y_{w(\infty)}^{\text {mat }}\right.$ and $\left.Y_{w(\infty)}^{\text {cav }}\right)$ separately. $Y_{w(\infty)}^{\text {mat }}$ was identified from experimental results in reference samples and $Y_{w(\infty)}^{c a v}$ could be obtained by calculating the amount of water needed to fill the volume of voids.

\section{Solubility determination of porous samples}

In "Fickian description of water sorption" section, water solubility in porous samples was identified from sorption curves, but no link to the porosity was established. To go further into the description, it should be possible to link the water mass fraction at equilibrium to the void fraction of each sample.

Equation (12) was proposed to calculate the equilibrium mass fraction of water in cavities. From Eqs. (8) and (12), the equilibrium total water mass fraction in samples could be expressed as in Eq. (15):

$Y_{\mathrm{w}(\infty)}=Y_{\mathrm{w}(\infty)}^{\mathrm{mat}}+\frac{f \rho_{\mathrm{w}}}{\rho_{\mathrm{m}}(1-f)}$,

where $Y_{\mathrm{w}(\infty)}^{\mathrm{mat}}$ is the water content at equilibrium of the matrix, which is the solubility of the reference material identified from experiments (see Fig. 13).

A closing of voids under high pressure was assumed to consider the reduction in equilibrium water content. A poro-mechanical model could link porosity and hydrostatic pressure and thus describe the compaction of cavities due to high pressure. An alternate way here was to introduce a compaction factor $(\gamma)$ of the initial porosity measured at atmospheric pressure; void fraction of samples could then be expressed as

$f=\gamma \cdot f_{0}$.

Compaction factor $\gamma$ is calculated from Eqs. (15) and (16) based on equilibrium water content of porous samples tested under high water pressure (see Fig. 13).

However, amounts of water calculated with Eq. (15) at atmospheric pressure $(\gamma=1)$ were considerably larger than equilibrium water contents previously identified in porous samples. In order to fit calculated solubility to values identified (see Fig. 13), a modified expression to calculate equilibrium water mass fraction of porous samples was proposed:
$Y_{\mathrm{w}(\infty)}=Y_{\mathrm{w}(\infty)}^{\mathrm{mat}}+K \frac{\gamma \cdot f_{0} \rho_{w}}{\rho_{\mathrm{m}}\left(1-\gamma \cdot f_{0}\right)}$.

$K$ is a partial saturation factor of voids at atmospheric pressure. This idea had already been proposed in previous works studying the effect of voids on water content in polymeric composites [15, 17]. This partial saturation of cavities could be explained by the fact that spatial distribution, shape, size or orientation of cavities can affect their accessibility to water.

Parameter $K$ was identified from equilibrium water content of porous samples at atmospheric pressure found in "Fickian description of water sorption" section (see Fig. 10). Since at atmospheric pressure there was no compaction of voids $(\gamma=1), K$ was the only unknown in Eq. (17).

Values of $K$ factor calculated for porous samples with 4.3 and $14 \%$ of void fraction are plotted in Fig. 15. A higher value of $K$ was found for samples with $14 \%$ of void fraction. This difference with samples with $4.3 \%$ of void fraction would suggest a difference of partial saturation level and thereby a difference of cavities morphology, in waisted and notched specimens.

Identified values of $\gamma$ are plotted in Fig. 15. Its evolution suggested that compressibility of porous samples was more significant in those with a higher void fraction (14\%).

In order to simulate the water uptake behaviour in porous samples with the proposed Langmuir-type sorption model, an element finite model was implemented.

\section{Finite element modelling (FEM) of water sorption}

An axisymmetric finite element model representing the cylindrical geometry of samples was implemented in Abaqus $^{\mathrm{TM}}$ software. Boundary condition at the surface was the water mass fraction at equilibrium in the matrix $Y_{\mathrm{w}(\infty)}^{\mathrm{mat}}$. Void fraction was considered to be homogenous in the sample and was included in the model as an internal variable. The mesh used in the model is presented in
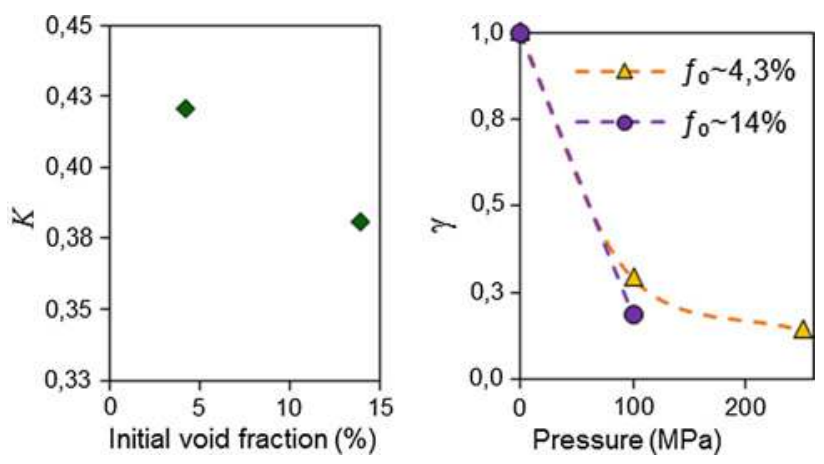

Fig. 15 Partial saturation coefficient $K$ and compaction of pores factor $\gamma$ identified to describe the effect of voids and pressure on the equilibrium water content of porous samples 
(a)

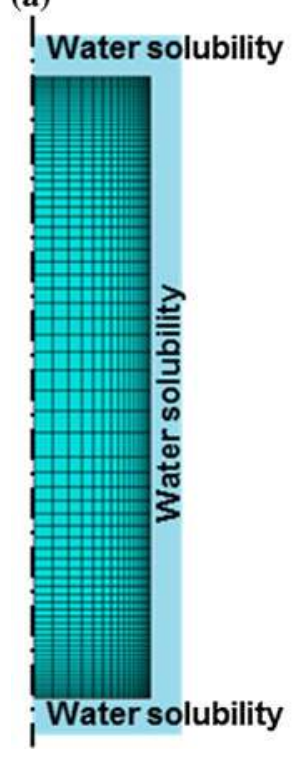

(b)

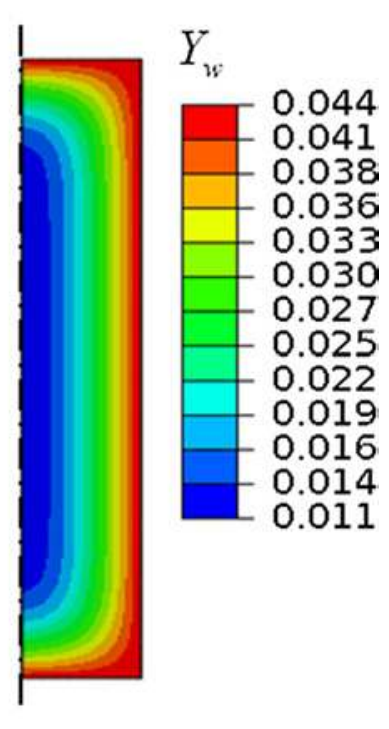

Fig. 16 a Axisymmetric FEM model for water diffusion simulation. b Contour plot of total water mass fraction $Y_{\mathrm{w}}$ calculated after $2000 \mathrm{~h}$ at atmospheric pressure for a porous sample with $14 \%$ of void fraction

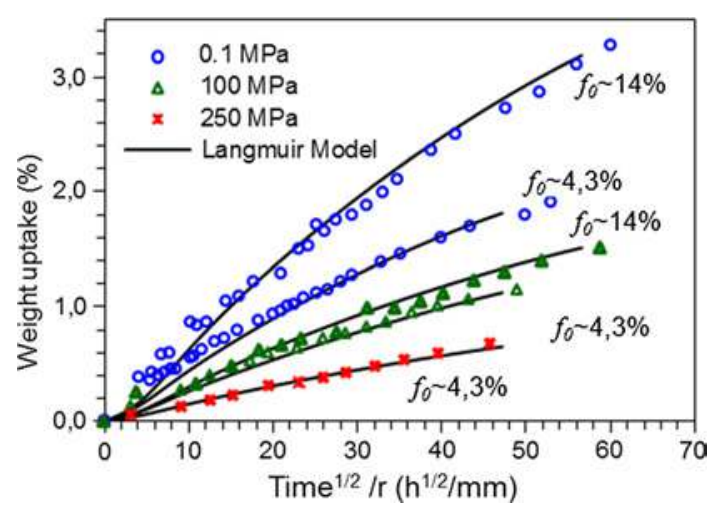

Fig. 17 Description of water uptake of porous samples with the proposed Langmuir-type sorption model

Fig. 16a and consists in linear elements which become finer and finer when close to the surface, where the size of elements reach $10 \mu \mathrm{m}$.
Water diffusion was simulated thanks to the transport solver of Abaqus ${ }^{\mathrm{TM}}$ with the HETVAL user subroutine, which allows the calculation of water fraction mass in cavities $Y_{w(\infty)}^{\text {cav }}$ by integrating the "sink" term (Eq. (13)) in the transport equation. Total water fraction mass is then calculated as a function of time and position at each integration point. Figure $16 \mathrm{~b}$ showed the contour plot of the total water mass fraction calculated with this model after $2000 \mathrm{~h}$ for a porous sample with $14 \%$ of void fraction immersed at atmospheric pressure.

Parameters $\alpha$ and $\tau$ were identified by finding the best fitting between experimental curves and theoretical curves obtained with the proposed Langmuir-type diffusion model.

Sorption curves obtained from this model are compared with experiments in Fig. 17, and Table 1 summarizes the full set of identified parameters.

Parameter $\alpha$ is a proportional factor that determined the global kinetics of water diffusion in porous samples and was found to increase with initial void fraction. With increasing values of $\alpha$, sink term in the transport equation (Eq. (13)) became preponderant and filling of voids slowed down the global diffusion kinetics. Moreover, $\alpha$ showed to be independent of hydrostatic pressure. That indicated a non-variable global diffusivity with increasing pressure, as observed with the classical Fick's model. Parameter $\tau$ represented a time constant which governs rate of the filling of voids and it was found to be independent of void fraction and hydrostatic pressure.

The advantage of the proposed Langmuir-type sorption model is the hypothesis of a sorption mechanism directly linked with the void fraction.

We showed that it is necessary to take into account the evolution of porosity under confinement for a correct modelling of water sorption in porous samples under hydrostatic pressure. The implementation of the proposed Langmuir-type sorption model in a FEM code enables to consider a coupling with a poro-mechanical model, thanks to the porosity which is associated to the volume change of the material. The space-time distribution of porosity could in fact be evaluated through a porous viscoplastic constitutive law which is the more appropriate to describe the mechanical behaviour of semicrystalline polymers.

Table 1 Identified parameters of the Langmuir-type sorption model implemented to describe water absorption behaviour of porous samples at several levels of hydrostatic pressure

\begin{tabular}{|c|c|c|c|c|c|c|c|c|}
\hline Initial void fraction & Pressure & $D^{\text {mat }}\left(10^{-10} \mathrm{~cm}^{2} / \mathrm{s}\right)$ & $Y_{\mathrm{w}(\infty)}^{\mathrm{mat}}$ & $Y_{\mathrm{w}(\infty)}^{\mathrm{cav}}$ & $\alpha$ & $\tau(\mathrm{h})$ & $K$ & $\gamma$ \\
\hline \multirow[t]{3}{*}{$4.3 \%$} & Atmospheric & 4.55 & $0.90 \mathrm{E}-2$ & $1.11 \mathrm{E}-2$ & 0.20 & 100 & 0.42 & 1.00 \\
\hline & $100 \mathrm{MPa}$ & & $0.49 \mathrm{E}-2$ & $0.73 E-2$ & 0.20 & 100 & 1.00 & 0.29 \\
\hline & $250 \mathrm{MPa}$ & & $0.33 \mathrm{E}-2$ & $0.35 \mathrm{E}-2$ & 0.20 & 100 & 1.00 & 0.14 \\
\hline \multirow[t]{2}{*}{$14 \%$} & Atmospheric & 4.55 & $0.90 \mathrm{E}-2$ & $3.64 \mathrm{E}-2$ & 0.70 & 100 & 0.38 & 1.00 \\
\hline & $100 \mathrm{MPa}$ & & $0.49 \mathrm{E}-2$ & $1.56 \mathrm{E}-2$ & 0.70 & 100 & 1.00 & 0.19 \\
\hline
\end{tabular}




\section{Conclusion}

The presented study includes experimental work and modelling which contributes to the understanding of the water sorption in a semicrystalline fluoropolymer grade. The originality of experiments deals with two principal aspects:

- The porous microstructure of the polymer due to a previously induced cavitation process.

- Characterization of water sorption under an extreme environment: high hydrostatic pressure (100 and $250 \mathrm{MPa})$.

Experimental study on the diffusion behaviour of the samples revealed that water content highly depends on the porosity and on the mechanical confining state of the material. Equilibrium water content was found to increase with the void fraction, whereas hydrostatic pressure had the opposite effect. Diffusivity was found to decrease with the void fraction and to be independent on the hydrostatic pressure.

Two sorption models were discussed to describe the water transport behaviour in the polymer: the classical Fick's theory showed to be in agreement with experimental results for reference and porous samples under several pressures. Furthermore, with the purpose of extending this characterization to the diffuso-mechanical behaviour of porous polymers, a Langmuir-type sorption model was proposed. For this model, it was assumed that the amount of water absorbed by porous samples could be related to its current porosity, which could be partially compacted under hydrostatic pressure. Conversely, additional parameters needed to be introduced in this model to obtain satisfactory agreements with experiments:

- $\alpha$ : water transfer coefficient between matrix and voids

- $\tau$ : characteristic time which rules the filling rate of voids

- $K$ : coefficient indicating partial saturation of voids at atmospheric pressure

- $\gamma$ : coefficient indicating compaction of voids under pressure

The direct link of the Langmuir-type sorption model to the porosity of the material provides a route to the construction of a poro-diffuso-mechanical model coupling the well-known viscoplastic behaviour of semicrystalline polymers with water diffusion under conditions of high pressure. Description of this diffuso-mechanical behaviour in porous polymers is the purpose of a following study.

Acknowledgements The authors would like to thank Aurélie Samouillet, Patrick Bourguelat, Karine Esterlé and Michel Chardin from IFP Energies Nouvelles for their technical assistance during experiments.

\section{References}

1. Filyanov E, Tarakanov O, Shamov I (1974) Effect of hydrostatic pressure on the water absorption of epoxy polymers. Polymer Mech 10:163-165

2. Le Gac P-Y, Davies P, Choqueuse D (2014) Evaluation of long term behaviour of polymers for offshore oil and gas applications. Oil Gas Sci Technol 70(2):279-289

3. Tucker W, Lee S, Rockett T (1993) The effects of pressure on water transport in polymers. J Compos Mater 27:756-763

4. Whitaker G, Darby M, Wostenholm G (1991) Influence of temperature and hydrostatic pressure on moisture absorption in polymer resins. J Mater Sci 26:49-55. doi:10.1007/BF00576031

5. Choqueuse D, Davies P, Mazeas F, Baizeau R (1997) Aging of composites in water: comparison of five materials in terms of absorption kinetics and evolution of mechanical properties. ASTM Spec Technol Publ 1302:73-96

6. Pollard A, Baggott R, Wostenholm G (1989) Influence of hydrostatic pressure on the moisture absorption of glass fibrereinforced polyester. J Mater Sci 24:1665-1669. doi:10.1007/ BF01105689

7. Neumann S, Marom G (1986) Free-volume dependent moisture diffusion under stress in composite materials. J Mater Sci 21:26-30. doi:10.1007/BF01144694

8. Avena A, Bunsell A (1988) Effect of hydrostatic pressure on the water absorption of glass fibre-reinforced epoxy resin. Composites. 19:355-357

9. Fahmy AA, Hurt JC (1980) Stress dependence of water diffusion in epoxy resin. Polym Compos 1:77-80

10. Neumann S, Marom G (1985) Stress dependence of the coefficient of moisture diffusion in composite materials. Polym Compos 6:9-12

11. Weitsman Y (1987) Stress assisted diffusion in elastic and viscoelastic materials. J Mech Phys Solids 35(1):73-94

12. Sar BE, Fréour S, Davies P, Jacquemin F (2012) Coupling moisture diffusion and internal mechanical states in polymers-a thermodynamical approach. Eur J Mech A 36:38-43

13. Gueribiz D, Jacquemin F, Fréour S (2013) A moisture diffusion coupled model for composite materials. Eur J Mech A 42:81-89

14. Harper B, Staab G, Chen R (1987) A note on the effects of voids upon the hygral and mechanical properties of AS4/3502 graphite/ epoxy. J Compos Mater 21:280-289

15. Thomason JL (1995) The interface region in glass fibre-reinforced epoxy resin composites: 2 . Water absorption, voids and the interface. Composites 26:477-485

16. Choi HS, Ahn KJ, Nam J, Chun HJ (2001) Hygroscopic aspects of epoxy/carbon ${ }^{\circledR}$ ber composite laminates in aircraft environments, 32

17. Costa ML, Rezende MC, de Almeida SFM (2006) Effect of void content on the moisture absorption in polymeric composites. Polymer Plast Technol Eng 45:691-698

18. Hedenqvist M, Ritums J, Condé-Brana M, Bergman G (2004) Structure and properties of poly (ethylene-co-chlorotrifluoroethylene) and polyvinylidene fluoride exposed to water, hydrochloric acid, hydrobromic acid and tetrachloroethylene. Polym Eng Sci 44:113-122

19. Hansen C (2001) Water transport and condensation in fluoropolymer films. Prog Org Coat 42:167-178

20. Bridgman PW (1944) The stress distribution at the neck of a tension specimen. Trans ASM 32:553-572 
21. Bridgman P (1947) The rheological properties of matter under high pressure. J Colloid Sci 2:7-16

22. Rosenberg E, Brusselle-Dupend N, Epsztein T (2011) A mesoscale quantification method of cavitation in semicrystalline polymers using X-ray microtomography. Mater Sci Eng A 528:6535-6544

23. Brusselle-Dupend N, Rosenberg E, Adrien J (2011) Characterization of cavitation development while tensile testing PVF2 using 3D X-ray microtomography. Mater Sci Eng A 530:36-50

24. Laiarinandrasana L, Morgeneyer TF, Proudhon H, N'guyen F, Maire E (2012) Effect of multiaxial stress state on morphology and spatial distribution of voids in deformed semicrystalline polymer assessed by X-ray tomography. Macromolecules 45:4658-4668

25. Rowe BW, Freeman BD, Paul DR, December RV, Re V, Recei M et al (2007) Effect of sorbed water and temperature on the optical properties and density of thin glassy polymer films on a silicon substrate. Mac romolecules 1:2806-2813
26. Fick A (1855) Ueber diffusion. Ann Phys 170:59-86

27. Crank J (1979) The mathematics of diffusion. Clarendon Press, Oxford

28. Wan YZ, Wang YL, Huang Y, He BM, Han KY (2005) Hygrothermal aging behaviour of VARTMed three-dimensional braided carbon-epoxy composites under external stresses. Compos A 36:1102-1109

29. Carter HG, Kibler KG (1978) Langmuir-type model for anomalous moisture diffusion in composite resins. J Compos Mater 12:118-131

30. Popineau S, Rondeau-Mouro C, Sulpice-Gaillet C, Shanahan MER (2005) Free/bound water absorption in an epoxy adhesive. Polymer 46:10733-10740

31. Leger R, Roy A, Grandidier JC (2010) Non-classical water diffusion in an industrial adhesive. Int $\mathrm{J}$ Adhes Adhes 30:744-753 